\title{
Solution of a Class of High Order Differential Equations
}

\author{
Weida Qin \\ School of Mathematics and Statistics \\ Hechi University \\ Yizhou, China \\ E-mail: qwd1983510@163.com
}

\author{
Wusheng Wang \\ School of Mathematics and Statistics \\ Hechi University \\ Yizhou, China \\ E-mail: wang4896@126.com
}

\begin{abstract}
In order to solve physical problems, we must establish mathematical models for the problems. Mathematical models often are differential equations relating an unknown function and one or more of its derivatives. In this paper, in order to solve high order differential equations, we first proved the expression for $n$ repeated definite integrals by mathematical induction, Integration by parts and binomial formula. Then we obtained the solution of a class of high order differential equations by integral technique and the formula for $n$ repeated definite integrals. Our results can be used to study the properties of high order differential equations, and then our results can be used to investigate physical or "real life" problems.
\end{abstract}

Keywords-Higher Order Differential Equation; Integration by Parts; Binomial Formula; Induction; Solution

\section{INTRODUCTION}

In order to solve physical problems, we must establish mathematical models for the problems. Mathematical models often are differential equations relating an unknown function and one or more of its derivatives. Wide applications of differential equations have attracted great interests of many mathematicians. Some mathematicians investigated existence of solutions for differential equations in [1-6]. Some mathematicians studied the solutions of high order differential equations and the theory on differential equations in [7-11].

\section{MAIN RESULTS}

In this section, we use the following lemmas to prove our main results.

Lemma 1. (see [12]) Suppose that $f(t)$ is a continuous function on $[a, \infty)$. Then the function

$$
\begin{aligned}
& y(t)=\int_{a}^{t} \int_{a}^{s} \int_{a}^{\tau_{1}} \cdots \int_{a}^{\tau_{n-1}} f\left(\tau_{n}\right)\left(d \tau_{n} \cdots d \tau_{2} d \tau_{1} d s\right) \\
& =\int_{a}^{t} \frac{(t-s)^{n}}{(n) !} f(s) d s,
\end{aligned}
$$

is a solution of higher order differential equation

$$
\left\{\begin{array}{l}
y^{(n+1)}(t)=f(t), t \in[a, \infty), \\
y^{(i)}(a)=0, i=0,1, \mathrm{~L}, n .
\end{array}\right.
$$

Proof. For the reader's convenience, we prove this Lemma by induction. Firstly, we consider the case of $n=1$. The solution of differential equation (2) is

$$
\begin{aligned}
y(t) & =\int_{a}^{t} \int_{a}^{s} f\left(\tau_{1}\right) d \tau_{1} d s \\
& =\left.s \int_{a}^{s} f\left(\tau_{1}\right) d \tau_{1}\right|_{a} ^{t}-\int_{a}^{t} s f(s) d s \\
& =\int_{a}^{t} f(t-s) f(s) d s .
\end{aligned}
$$

So (1) is true for $n=1$. Letting this be our basis step, suppose that (1) is true up to some $k>1$, i.e. the solution of differential equation (2) is

$$
\begin{aligned}
y(t) & =\int_{a}^{t} \int_{a}^{s} \int_{a}^{\tau_{1}} \cdots \int_{a}^{\tau_{k-1}} f\left(\tau_{k}\right)\left(d \tau_{k} \cdots d \tau_{2} d \tau_{1} d s\right) \\
& =\int_{a}^{t} \frac{(t-s)^{k}}{(k) !} f(s) d s
\end{aligned}
$$

If $n=k+1$, then the induction hypothesis implies that the solution of differential equation (2) is

$$
\begin{aligned}
& y(t)=\int_{a}^{t} \int_{a}^{s} \int_{a}^{\tau_{1}} \cdots \int_{a}^{\tau_{k}} f\left(\tau_{k+1}\right)\left(d \tau_{k+1} \cdots d \tau_{2} d \tau_{1} d s\right) \\
& =\int_{a}^{t} \int_{a}^{s} \frac{\left(s-\tau_{1}\right)^{k}}{(k) !} f\left(\tau_{1}\right) d \tau_{1} d s . \\
& =\left.s \int_{a}^{s} \frac{\left(s-\tau_{1}\right)^{k}}{(k) !} f\left(\tau_{1}\right) d \tau_{1}\right|_{a} ^{t} \\
& \quad-\int_{a}^{t} s \int_{a}^{s} \frac{\left(s-\tau_{1}\right)^{k-1}}{(k-1) !} f\left(\tau_{1}\right) d \tau_{1} d s \\
& =t \int_{a}^{t} \frac{(t-s)^{k}}{(k) !} f(s) d s-\left(\left.\frac{s^{2}}{2} \int_{a}^{s} \frac{\left(s-\tau_{1}\right)^{k-1}}{(k-1) !} f\left(\tau_{1}\right) d \tau_{1}\right|_{a} ^{t}\right. \\
& \left.\quad-\int_{a}^{t} \frac{s^{2}}{2} \int_{a}^{s} \frac{\left(s-\tau_{1}\right)^{k-2}}{(k-2) !} f\left(\tau_{1}\right) d \tau_{1} d s\right) \\
& =t \int_{a}^{t} \frac{(t-s)^{k}}{(k) !} f(s) d s-\left(\frac{t^{2}}{2} \int_{a}^{t} \frac{(t-s)^{k-1}}{(k-1) !} f(s) d s\right. \\
& \left.\quad-\int_{a}^{t} \frac{s^{2}}{2} \int_{a}^{s} \frac{\left(s-\tau_{1}\right)^{k-2}}{(k-2) !} f\left(\tau_{1}\right) d \tau_{1} d s\right) .
\end{aligned}
$$


We consider the integration

$\int_{a}^{t} \frac{s^{2}}{2} \int_{a}^{s} \frac{\left(s-\tau_{1}\right)^{k-2}}{(k-2) !} f\left(\tau_{1}\right) d \tau_{1} d s$.

(6)

When $k-2=1$, we have

$\int_{a}^{t} \frac{s^{2}}{2} \int_{a}^{s}\left(s-\tau_{1}\right) f\left(\tau_{1}\right) d \tau_{1} d s$

$=\left.\frac{s^{3}}{3 !} \int_{a}^{s}\left(s-\tau_{1}\right) f\left(\tau_{1}\right) d \tau_{1}\right|_{a} ^{t}-\int_{a}^{t} \frac{s^{3}}{3 !} \int_{a}^{s} f\left(\tau_{1}\right) d \tau_{1} d s$

$=\frac{t^{3}}{3 !} \int_{a}^{t}(t-s) f(s) d s$

$-\left(\left.\frac{s^{4}}{4 !} \int_{a}^{s} f\left(\tau_{1}\right) d \tau_{1}\right|_{a} ^{t}-\int_{a}^{t} \frac{s^{4}}{4 !} f(s) d s\right)$

$=\frac{t^{3}}{3 !} \int_{a}^{t}(t-s) f(s) d s-\frac{t^{4}}{4 !} \int_{a}^{t} f(s) d s+\int_{a}^{t} \frac{s^{4}}{4 !} f(s) d s$

$=\int_{a}^{t} \frac{\frac{4 !}{3 ! !} t^{3}(t-s)-t^{4}+s^{4}}{4 !} f(s) d s$.

When $k-2=2$, we have

$\int_{a}^{t} \frac{s^{2}}{2} \int_{a}^{s} \frac{\left(s-\tau_{1}\right)^{2}}{2 !} f\left(\tau_{1}\right) d \tau_{1} d s$

$=\left.\frac{s^{3}}{3 !} \int_{a}^{s} \frac{\left(s-\tau_{1}\right)^{2}}{2 !} f\left(\tau_{1}\right) d \tau_{1}\right|_{a} ^{t}$

$-\int_{a}^{t} \frac{s^{3}}{3 !} \int_{a}^{s}\left(s-\tau_{1}\right) f\left(\tau_{1}\right) d \tau_{1} d s$

$=\frac{t^{3}}{3 !} \int_{a}^{t} \frac{(t-s)^{2}}{2 !} f(s) d s$

$-\left[\left.\frac{s^{4}}{4 !} \int_{a}^{s}\left(s-\tau_{1}\right) f\left(\tau_{1}\right) d \tau_{1}\right|_{a} ^{t}-\int_{a}^{t} \frac{s^{4}}{4 !} \int_{a}^{s} f\left(\tau_{1}\right) d \tau_{1} d s\right]$

$=\frac{t^{3}}{3 !} \int_{a}^{t} \frac{(t-s)^{2}}{2 !} f(s) d s-\left\{\left.\frac{s^{4}}{4 !} \int_{a}^{s}\left(s-\tau_{1}\right) f\left(\tau_{1}\right) d \tau_{1}\right|_{a} ^{t}\right.$

$\left.-\left[\left.\frac{s^{5}}{5 !} \int_{a}^{s} f\left(\tau_{1}\right) d \tau_{1}\right|_{a} ^{t}-\int_{a}^{t} \frac{s^{5}}{5 !} f(s) d s\right]\right\}$

$=\frac{t^{3}}{3 !} \int_{a}^{t} \frac{(t-s)^{2}}{2 !} f(s) d s-\left\{\frac{t^{4}}{4 !} \int_{a}^{t}(t-s) f(s) d s\right.$

$\left.-\left[\frac{t^{5}}{5 !} \int_{a}^{t} f(s) d s-\int_{a}^{t} \frac{s^{5}}{5 !} f(s) d s\right]\right\}$

$=\int_{a}^{t} \frac{\frac{5 !}{3 ! 2 !} t^{3}(t-s)^{2}-\frac{5 !}{4 ! !} t^{4}(t-s)+t^{5}-s^{5}}{5 !} f(s) d s$.

When $k-2=3$, we get

$\int_{a}^{t} \frac{s^{2}}{2} \int_{a}^{s} \frac{\left(s-\tau_{1}\right)^{3}}{3 !} f\left(\tau_{1}\right) d \tau_{1} d s$

$$
\begin{aligned}
& =\left.\frac{s^{3}}{3 !} \int_{a}^{s} \frac{\left(s-\tau_{1}\right)^{3}}{3 !} f\left(\tau_{1}\right) d \tau_{1}\right|_{a} ^{t} \\
& -\int_{a}^{t} \frac{s^{3}}{3 !} \int_{a}^{s} \frac{\left(s-\tau_{1}\right)^{2}}{2 !} f\left(\tau_{1}\right) d \tau_{1} d s \\
& =\frac{t^{3}}{3 !} \int_{a}^{t} \frac{(t-s)^{3}}{3 !} f(s) d s \\
& -\left[\left.\frac{s^{4}}{4 !} \int_{a}^{s} \frac{\left(s-\tau_{1}\right)^{2}}{2 !} f\left(\tau_{1}\right) d \tau_{1}\right|_{a} ^{t}\right. \\
& \left.-\int_{a}^{t} \frac{s^{4}}{4 !} \int_{a}^{s}\left(s-\tau_{1}\right) f\left(\tau_{1}\right) d \tau_{1} d s\right] \\
& =\frac{t^{3}}{3 !} \int_{a}^{t} \frac{(t-s)^{3}}{3 !} f(s) d s-\left\{\left.\frac{s^{4}}{4 !} \int_{a}^{s} \frac{\left(s-\tau_{1}\right)^{2}}{2 !} f\left(\tau_{1}\right) d \tau_{1}\right|_{a} ^{t}\right. \\
& \left.-\left[\left.\frac{s^{5}}{5 !} \int_{a}^{s}\left(s-\tau_{1}\right) f\left(\tau_{1}\right) d \tau_{1}\right|_{a} ^{t}-\int_{a}^{t} \frac{s^{5}}{5 !} \int_{a}^{s} f\left(\tau_{1}\right) d \tau_{1} d s\right]\right\} \\
& =\frac{t^{3}}{3 !} \int_{a}^{t} \frac{(t-s)^{3}}{3 !} f(s) d s-\left\{\frac{t^{4}}{4 !} \int_{a}^{t} \frac{(t-s)^{2}}{2 !} f(s) d s\right. \\
& \left.-\left[\frac{t^{5}}{5 !} \int_{a}^{t}(t-s) f\left(\tau_{1}\right) d s-\left(\frac{t^{6}}{6 !} \int_{a}^{t} f(s) d s-\int_{a}^{t} \frac{s^{6}}{6 !} f(s) d s\right)\right]\right\} \\
& =\frac{1}{6 !} \int_{a}^{t}\left(\frac{6 !}{3 ! 3 !} t^{3}(t-s)^{3}-\frac{6 !}{4 ! 2 !} t^{4}(t-s)^{2}\right) f(s) d s \text {. } \\
& +\frac{1}{6 !} \int_{a}^{t}\left(\frac{6 !}{5 ! 1 !} t^{5}(t-s)-t^{6}+s^{6}\right) f(s) d s
\end{aligned}
$$

By the same procedure as above, we get$$
\int_{a}^{t} \frac{s^{2}}{2} \int_{a}^{s} \frac{\left(s-\tau_{1}\right)^{m-2}}{(m-2) !} f\left(\tau_{1}\right) d \tau_{1} d s
$$$$
=\frac{t^{3}}{3 !} \int_{a}^{t} \frac{(t-s)^{m-2}}{(m-2) !} f(s) d s
$$$$
+(-1)^{3} \frac{t^{4}}{4 !} \int_{a}^{t} \frac{(t-s)^{m-3}}{(m-3) !} f(s) d s+\cdots
$$$$
+(-1)^{m-2} \frac{t^{m-1}}{(m-1) !} \int_{a}^{t} \frac{(t-s)^{2}}{2 !} f(s) d s
$$$$
+(-1)^{m-1} \frac{t^{m}}{m !} \int_{a}^{t}(t-s) f(s) d s
$$$$
+(-1)^{m} \frac{t^{m+1}}{(m+1) !} \int_{a}^{t} f(s) d s+(-1)^{m+1} \int_{a}^{t} \frac{s^{m+1}}{(m+1) !} f(s) d s
$$$$
=\frac{1}{(m+1) !} \int_{a}^{t}\left[\frac{(m+1) !}{3 !(m-2) !} t^{3}(t-s)^{m-2}\right.
$$$$
-\frac{(m+1) !}{4 !(m-3) !} t^{4}(t-s)^{m-3}+\cdots
$$ 


$$
\begin{aligned}
& +(-1)^{m-2} \frac{(m+1) !}{(m-1) ! 2 !} t^{m-1}(t-s)^{2} \\
& +(-1)^{m-1} \frac{(m+1) !}{m ! !} t^{m}(t-s)+(-1)^{m} t^{m+1} \\
& \left.\quad+(-1)^{m+1} s^{m+1}\right] f(s) d s .
\end{aligned}
$$

Taking into account (5) and (10), we obtain

$$
\begin{gathered}
y(t)=\int_{a}^{t} \int_{a}^{s} \int_{a}^{\tau_{1}} \cdots \int_{a}^{\tau_{k}} f\left(\tau_{k+1}\right)\left(d \tau_{k+1} \cdots d \tau_{2} d \tau_{1} d s\right) \\
=t \int_{a}^{t} \frac{(t-s)^{k}}{k !} f(s) d s-\left(\frac{t^{2}}{2} \int_{a}^{t} \frac{(t-s)^{k-1}}{(k-1) !} f(s) d s\right. \\
\left.\quad-\int_{a}^{t} \frac{s^{2}}{2} \int_{a}^{s} \frac{\left(s-\tau_{1}\right)^{k-2}}{(k-2) !} f\left(\tau_{1}\right) d \tau_{1} d s\right) \\
=t \int_{a}^{t} \frac{(t-s)^{k}}{k !} f(s) d s-\left(\frac{t^{2}}{2} \int_{a}^{t} \frac{(t-s)^{k-1}}{(k-1) !} f(s) d s\right. \\
-\frac{1}{(k+1) !} \int_{a}^{t}\left[\frac{(k+1) !}{3 !(k-2) !} t^{3}(t-s)^{k-2}-\frac{(k+1) !}{4 !(k-3) !} t^{4}(t-s)^{k-3}\right. \\
+\cdots+(-1)^{k-2} \frac{(k+1) !}{(k-1) ! 2 !} t^{k-1}(t-s)^{2} \\
+(-1)^{k-1}(k+1) t^{k}(t-s)+(-1)^{k} t^{k+1}
\end{gathered}
$$$$
\left.\left.+(-1)^{k} t^{k+1}+(-1)^{k+1} s^{k+1}\right] f(s) d s\right)
$$$$
=\frac{1}{(k+1) !} \int_{a}^{t}\left[\frac{(k+1) !}{1 ! k !} t(t-s)^{k}-\frac{(k+1) !}{2 !(k-1) !} t^{2}(t-s)^{k-1}\right.
$$$$
+\cdots+(-1)^{k-2} \frac{(k+1) !}{(k-1) ! 2 !} t^{k-1}(t-s)^{2}
$$$$
+(-1)^{k-1}(k+1) t^{k}(t-s)
$$$$
\left.+(-1)^{k} t^{k+1}+(-1)^{k+1} s^{k+1}\right] f(s) d s
$$$$
=\frac{1}{(k+1) !} \int_{a}^{t}\left[-(t-s)^{k+1}+\frac{(k+1) !}{1 ! k !} t(t-s)^{k}\right.
$$$$
-\frac{(k+1) !}{2 !(k-1) !} t^{2}(t-s)^{k-1}
$$$$
+\cdots+(-1)^{k-2} \frac{(k+1) !}{(k-1) ! 2 !} t^{k-1}(t-s)^{2}
$$$$
+(-1)^{k-1}(k+1) t^{k}(t-s)+(-1)^{k} t^{k+1}
$$$$
\left.\left.+(t-s)^{k+1}+(-1)^{k+1} s^{k+1}\right] f(s) d s\right)
$$$$
=\int_{a}^{t}\left[\frac{-(-s)^{k+1}+(t-s)^{k+1}+(-1)^{k+1} s^{k+1}}{(k+1) !}\right] f(s) d s
$$$$
=\int_{a}^{t} \frac{(t-s)^{k+1}}{(k+1) !} f(s) d s .
$$

Therefore, by induction, (17) is true for all $n>1$.

Theorem 1. Suppose that $f(t)$ is a continuous function on $[a, \infty)$, and $p(t)>0$ is an nth continuous differentiable function on $[a, \infty)$. Then the function $y(t)=\int_{a}^{t} \frac{(t-s)^{m-1}}{(m-1) !}\left(\frac{1}{p(s)} \int_{a}^{s} \frac{(s-\tau)^{n-1}}{(n-1) !} f(\tau) d \tau\right) d s$

is a solutions of higher order differential equation

$\left\{\begin{array}{l}\frac{d^{n}}{d t^{n}}\left(p(t) \frac{d^{m}}{d t^{m}} y(t)\right)=f(t), t \in[a, \infty), \\ y^{(i)}(a)=0, i=0,1, \cdots, n+m-1 .\end{array}\right.$

Proof. Carrying out integrations on both sides of (13) $n$ times, using (1), we obtain

$$
\begin{gathered}
p(t) \frac{d^{m}}{d t^{m}} y(t)=\int_{a}^{t} \int_{a}^{s} \int_{a}^{\tau_{1}} \mathrm{~L} \int_{a}^{\tau_{n-2}} f\left(\tau_{n-1}\right)\left(d \tau_{n-1} \mathrm{~L} d \tau_{2} d \tau_{1} d s\right) \\
=\int_{a}^{t} \frac{(t-s)^{n-1}}{(n-1) !} f(s) d s .
\end{gathered}
$$

Dividing both sides of (14) by $p(t)$, we have

$\frac{d^{m}}{d t^{m}} y(t)=\frac{1}{p(t)} \int_{a}^{t} \frac{(t-s)^{n-1}}{(n-1) !} f(s) d s$.

From (15), we get

$$
y(t)=\int_{a}^{t} \frac{(t-s)^{m-1}}{(m-1) !}\left(\frac{1}{p(s)} \int_{a}^{s} \frac{(s-\tau)^{n-1}}{(n-1) !} f(\tau) d \tau\right) d s .
$$

(16) is a solution of higher order differential equation (13).

Theorem 2. Suppose that $c_{1}, c_{2}$ are positive constants. Then the function

$$
y(t)=\frac{c_{1}(t-a)^{m+n}}{c_{2}(m+n) !}
$$

is a solutions of higher order differential equation

$\left\{\begin{array}{l}\frac{d^{n}}{d t^{n}}\left(c_{2} \frac{d^{m}}{d t^{m}} y(t)\right)=c_{1}, t \in[a, \infty) \\ y^{(i)}(a)=0, i=0,1, \cdots, n+m-1 .\end{array}\right.$

Proof. By Theorem 1, we see that the function

$y(t)=\frac{c_{1}}{c_{2}} \int_{a}^{t} \frac{(t-s)^{m-1}}{(m-1) !}\left(\int_{a}^{s} \frac{(s-\tau)^{n-1}}{(n-1) !} d \tau\right) d s$

is a solutions of higher order differential equation

$$
\left\{\begin{array}{l}
\frac{d^{n}}{d t^{n}}\left(c_{2} \frac{d^{m}}{d t^{m}} y(t)\right)=c_{1}, t \in[a, \infty), \\
y^{(i)}(a)=0, i=0,1, \cdots, n+m-1 .
\end{array}\right.
$$

From (14), we have

$$
\begin{aligned}
y(t) & =\frac{c_{1}}{c_{2}} \int_{a}^{t} \frac{(t-s)^{m-1}}{(m-1) !}\left(\int_{a}^{s} \frac{(s-\tau)^{n-1}}{(n-1) !} d \tau\right) d s \\
& =\frac{c_{1}}{c_{2}} \int_{a}^{t} \frac{(t-s)^{m-1}}{(m-1) !}\left(-\left.\frac{(s-\tau)^{n}}{n !}\right|_{a} ^{s}\right) d s \\
& =\frac{c_{1}}{c_{2}} \int_{a}^{t} \frac{(t-s)^{m-1}(s-a)^{n}}{(m-1) ! n !} d s
\end{aligned}
$$




$$
=\frac{c_{1}}{c_{2}} \int_{a}^{t} \frac{(t-s)^{m-1}(s-a)^{n}}{(m-1) ! n !} d s .
$$

Let $s-a=x(t-a)$, then $t-s=t-a-(s-a)$.

From (21), we have

$$
\begin{aligned}
y(t) & =\frac{c_{1}(t-a)^{m+n}}{c_{2}} \int_{0}^{1} \frac{(1-v)^{m-1} v^{n}}{(m-1) ! n !} d v \\
& =\frac{c_{1}(t-a)^{m+n}}{(m-1) 1 n ! c_{2}} B(m, n+1) \\
& =\frac{c_{1}(t-a)^{m+n} n !(m-1) !}{(m-1) ! n !(m+n) ! c_{2}} \\
& =\frac{c_{1}(t-a)^{m+n}}{c_{2}(m+n) !} .
\end{aligned}
$$

\section{CONCLUSIONS}

In this paper, firstly, we proved the function

$$
\begin{aligned}
y(t) & =\int_{a}^{t} \int_{a}^{s} \int_{a}^{\tau_{1}} \mathrm{~L} \int_{a}^{\tau_{n-1}} f\left(\tau_{n}\right)\left(d \tau_{n} \mathrm{~L} d \tau_{2} d \tau_{1} d s\right) \\
= & \int_{a}^{t} \frac{(t-s)^{n}}{(n) !} f(s) d s
\end{aligned}
$$

be a solution of higher order differential equation

$$
\left\{\begin{array}{l}
y^{(n+1)}(t)=f(t), t \in[a, \infty), \\
y^{(i)}(a)=0, i=0,1, \mathrm{~L}, n .
\end{array}\right.
$$

Then we proved the function

$$
y(t)=\int_{a}^{t} \frac{(t-s)^{m-1}}{(m-1) !}\left(\frac{1}{p(s)} \int_{a}^{s} \frac{(s-\tau)^{n-1}}{(n-1) !} f(\tau) d \tau\right) d s
$$

be a solutions of higher order differential equation

$$
\left\{\begin{array}{l}
\frac{d^{n}}{d t^{n}}\left(p(t) \frac{d^{m}}{d t^{m}} y(t)\right)=f(t), t \in[a, \infty), \\
y^{(i)}(a)=0, i=0,1, \cdots, n+m-1 .
\end{array}\right.
$$

Finally, we proved the function

$$
y(t)=\frac{c_{1}(t-a)^{m+n}}{c_{2}(m+n) !}
$$

to be a solutions of higher order differential equation $\left\{\begin{array}{l}\frac{d^{n}}{d t^{n}}\left(c_{2} \frac{d^{m}}{d t^{m}} y(t)\right)=c_{1}, t \in[a, \infty) \\ y^{(i)}(a)=0, i=0,1, \cdots, n+m-1 .\end{array}\right.$

\section{ACKNOWLEDGMENT}

This research was supported by Guangxi Natural Science Foundation (No. 2012GXNSFAA053009) and Scientific Research Foundation of the Education Department of Guangxi Autonomous Region of China (No. KY2015ZD103, KY2015YB257), and the high school specialty and curriculum integration project of Guangxi Zhuang Autonomous Region (No. GXTSZY2220).

\section{REFERENCES}

[1] L. Guo and X. Zhang, "Existence of positive solutions for the singular fractional differential equations," J. Appl. Math. Comput., vol. 44, 2014, pp. 215-228.

[2] Y. Wang and L. Liu, "Necessary and sufficient condition for the existence of positive solution to singular fractional differential equations," Advances in Difference Equations, vol. 2015(207), 2015, pp. 1-14, DOI 10.1186/s13662-015-0540-5.

[3] W. Jiang, X. Huang, W. Guo and Q. Zhang, "The existence of positive solutions for the singular fractional differential equation," Journal of Applied Mathematics and Computing, vol. 41(1), March 2013, pp. 171-182.

[4] Z. Bai and T. Qiu, "Existence of positive solution for singular fractional differential equation," Applied Mathematics and Computation, vol. 215, 2009, pp. 2761-2767.

[5] S. Zhang, "Positive solutions to singular boundary value problem for nonlinear fractional differential equation," Computers and Mathematics with Applications, vol. 59, 2010, pp. 1300-1309.

[6] M. Naceri, R. Agarwal, E. Çetin and E. Amir, "Existence of solutions to fourth-order differential equations with deviating arguments," Boundary Value Problems, vol. 2015(108), 2015, pp. 1-31, DOI 10.1186/s13661-015-0373-X.

[7] J. Dzurina, B. Baculıkova, I. Jadlovsk, "Kneser solutions of fourthorder trinomial delay differential equations," Applied Mathematics Letters, vol. 49, 2015, pp. 67-72.

[8] X. He, S. Zheng, Global boundedness of solutions in a reactiondiffusion system of predator-prey model with prey-taxis, Applied Mathematics Letters, vol. 49, 2015, pp. 73-77.

[9] J. K. Hale, Odinary Differential Equation, Dover Publications, New Y ork, 2009.

[10] W. Zhang, Z. Du and B. Xu, Odinary Differential Equation(Higher Education Press, Beijing, 2013.

[11] M. T. Holm, The Theory of Discrete Fractional Calculus: Development and Application, PhD dissertation[D], University of Nebraska-Lincoln, 2011.

[12] I. Podlubny, Fractional Differential Equations, Academic Press, New York, 1999. 